\title{
Safety and outcomes of resection of butterfly glioblastoma
}

\author{
Fara Dayani, BS, ${ }^{1}$ Jacob S. Young, MD, ${ }^{2}$ Alexander Bonte, BA, ${ }^{3}$ Edward F. Chang, MD, PhD, ${ }^{2}$ \\ Philip Theodosopoulos, MD, ${ }^{2}$ Michael W. McDermott, MD, ${ }^{2}$ Mitchel S. Berger, MD, ${ }^{2}$ and \\ Manish K. Aghi, MD, PhD² \\ ${ }^{1}$ University of California, San Francisco School of Medicine; '2Department of Neurosurgery, University of California, San \\ Francisco, California; and ${ }^{3}$ Royal College of Surgeons in Ireland School of Medicine, Dublin, Ireland
}

\begin{abstract}
OBJECTIVE Butterfly glioblastoma (bGBM) is a rare type of GBM, characterized by a butterfly pattern on MRI studies because of its bihemispheric involvement and invasion of the corpus callosum (CC). There is a profound gap in the knowledge regarding the optimal treatment approach as well as the safety and survival benefits of resection in treating this aggressive brain tumor. In this retrospective study, authors add to our understanding of these tumors by identifying the clinical characteristics and outcomes of patients with bGBM.
\end{abstract}

METHODS An institutional database was reviewed for GBM cases treated in the period from 2004 to 2014. Records were reviewed to identify adult patients with bGBM. Cases of GBM with invasion of the CC without involvement of the contralateral hemisphere and bilateral GBMs without involvement of the CC were excluded from the study. Patient and tumor characteristics were gleaned from the medical records, and volumetric analysis was performed using T1-weighted MRI studies.

RESULTS From among 1746 cases of GBM, 39 cases of bGBM were identified. Patients had a mean age of 57.8 years at diagnosis. Headache and confusion were the most common presenting symptoms ( $48.7 \%$ and $33.3 \%$, respectively). The median overall survival was 3.2 months from diagnosis with an overall 6 -month survival rate of $38.1 \%$. Age, Karnofsky Performance Status at diagnosis, preoperative tumor volume, postoperative tumor volume, and extent of resection were found to significantly impact survival in the univariate analysis. On multivariate analysis, preoperative tumor volume and treatment approach of resection versus biopsy were identified as independent prognostic factors regardless of the patient-specific characteristics of age and KPS at diagnosis. Resection and biopsy were performed in $35.9 \%$ and $64.1 \%$ of patients, respectively. Resection was found to confer a better prognosis than biopsy (HR 0.37, $p=0.009$ ) with a minimum extent of resection of $86 \%$ to observe survival benefits (HR $0.054, p=0.03$ ). The rate of persistent neurological deficits from resection was $7.14 \%$. Patients younger than 70 years had a better prognosis (HR 0.32, $p=0.003$ ). Patients undergoing resection and receiving adjuvant chemoradiation had a better prognosis than patients who lacked one of the three treatment modalities ( $\mathrm{HR}=0.34, \mathrm{p}=0.015)$.

CONCLUSIONS Resection of bGBM is associated with low persistent neurological deficits, with improvement in survival compared to biopsy. A more aggressive treatment approach involving aggressive resection and adjuvant chemoradiation has significant survival benefits and improves outcome.

https://thejns.org/doi/abs/10.3171/2018.3.FOCUS1857

KEYWORDS butterfly glioblastoma; corpus callosum glioblastoma; resection

$\mathrm{B}$ UTTERFLY glioblastomas (bGBMs), which invade and cross the corpus callosum (CC) or interhemispheric commissure to involve the contralateral hemisphere, are thought to have extremely poor outcomes and are generally considered to be lesions for which the risks of resection outweigh the benefits. ${ }^{8}$ This tenet stems from the belief that these tumors represent a more diffuse and aggressive phenotype of an already aggressive and incurable disease. ${ }^{12}$ Moreover, in the past, patients who underwent resection have often suffered from significant

ABBREVIATIONS bGBM = butterfly glioblastoma; $C C=$ corpus callosum; $E O R=$ extent of resection; $K P S=$ Karnofsky Performance Status . ACCOMPANYING EDITORIAL DOI: 10.3171/2018.3.FOCUS18152.

SUBMITTED February 1, 2018. ACCEPTED March 28, 2018.

INCLUDE WHEN CITING DOI: 10.3171/2018.3.FOCUS1857. 
morbidity such as abulia or akinetic mutism. Accordingly, most patients undergo biopsy to confirm diagnosis and proceed with palliative medical and radiation treatment. The absence of objective data available to physicians has led to variable treatment approaches for the management of butterfly glioma, often excluding tumor resection. ${ }^{14}$

However, the aforementioned beliefs regarding the resection of butterfly glioma are largely based on case reports or small case series. ${ }^{1,3,15}$ Also, the conservative approach has left patients with significant bifrontal tumor burden and associated peritumoral edema, resulting in an extremely poor quality of life-with most patients quickly progressing to abulia. Moreover, resection of high-grade gliomas has been repeatedly demonstrated to improve symptom burden, mass effect from the lesion as well as the associated peritumoral edema, tumor control, and overall survival. ${ }^{2,5,13}$ Therefore, taking into account the natural history of the disease and the potential improvement that resection offers, the risk-benefit ratio of resection may, in fact, be tilted in favor of resection.

Although there is a paucity of research in this area, one study by Chaichana et al. compared 48 patients with bGBM to patients with GBM in other locations and found that having a lesion that crossed the $\mathrm{CC}$ was independently associated with a worse prognosis, with an overall survival of only 4.2 months for patients who underwent biopsy only. ${ }^{7}$ Importantly, the authors found that for patients with bGBM, resection improved survival and a more extensive tumor resection (at least $65 \%$ of the tumor) was associated with better overall survival. More recently, other authors have reported much more aggressive resections, with the extent of resection (EOR) better than $98 \%$ for most patients and a similar improvement in patient survival. ${ }^{6,11}$ One question that remains unanswered by these studies is whether the portion of tumor that invades the $\mathrm{CC}$ is important to resect or if the EOR achieved by removing the majority of tumor cells from the cerebral hemisphere is sufficient to improve overall survival.

In this study, we aimed to add to the understanding of these tumors by identifying the clinical characteristics of and outcomes for patients with bGBM at our institution. Currently, the therapeutic approach for bGBM is variable, with a lack of objective data available to providers.

\section{Methods \\ Study Population}

An institutional database (UCSF Medical Center) was reviewed for GBM cases treated in the period from 2004 to 2014. Diagnosis of GBM was verified via pathology report generated by our institution. A retrospective chart review was performed to identify adult patients (age $\geq 18$ years) with butterfly glioma, characterized as contrast-enhancing GBM invading bilateral hemispheres and the $\mathrm{CC}$ on imaging studies (CT and MRI). Cases of GBM with invasion of the $\mathrm{CC}$ without involvement of the contralateral hemisphere and bilateral GBMs without invasion of the CC were excluded from our analysis. This study was granted approval by our institutional review board. Since this was a retrospective chart review involving de-identified patient data, patient consent was not required for this study.

\section{Clinical Variables}

All patients had comprehensive documentation in their medical records including a presurgical exam (history and physical), preoperative imaging, operative report, postoperative imaging, and pathology report. With this information, we aimed to identify any patient-specific (age, sex, presenting symptoms, and functional status), tumor-specific (tumor location, tumor volume, and molecular/genetic characteristics), and treatment-specific (treatment modalities and EOR) factors. The Karnofsky Performance Status (KPS) score was used to assess a patient's functional status at different time points. The KPS score was either stated by the provider or retrospectively assigned by one of the authors (F.D.) based on the medical records.

\section{Volumetric Analysis}

Volumetric analysis of all cases was performed using Brainlab Smart Brush, a web-based software that calculates tumor volume based on 3D reconstructions of the tumor. For patients who had undergone tumor resection, volumetric analysis of their most recent preoperative scans and immediate postoperative scans was performed to determine EOR. The EOR was defined as (preoperative tumor volume - postoperative residual tumor volume)/ preoperative total tumor volume. All volumetric analyses were performed on postgadolinium T1-weighted MRI studies. The T1-weighted images were used to ensure that the blood products and postoperative changes were not considered as residual tumor on postoperative scans.

\section{Statistical Analysis}

All statistical analyses were performed using SPSS version 23 software (SPSS Inc.). A p value $\leq 0.05$ was considered statistically significant. The primary statistical tests performed for intergroup comparisons were Fisher's exact test for categorical data, the t-test for continuous parametric data, and the Mann-Whitney U-test for continuous nonparametric data. We also performed Kaplan-Meier survival analysis, log-rank test for survival curve comparison, univariate regression, and Cox regression analysis.

\section{Results}

We reviewed 1746 cases of GBM and identified 39 cases of bGBM that met our inclusion criteria, accounting for $2.2 \%$ of all GBMs in our study (Fig. 1A-D). Four cases (10.3\%) in our cohort were diagnosed during 2003 and 2004, before temozolomide became the standard of care in $2005 .^{16}$

\section{Patient Characteristics}

The clinical characteristics of all bGBM cases are summarized in Table 1. The mean age at diagnosis was $57.8 \pm$ 2.6 years with most patients presenting in their sixth decade of life. Male patients made up 59.0\% of the cohort, with female patients accounting for the remaining $41.0 \%$. The most common presenting symptoms included headache, cognitive decline including confusion and memory loss, and seizure. At the time of diagnosis, the majority of 

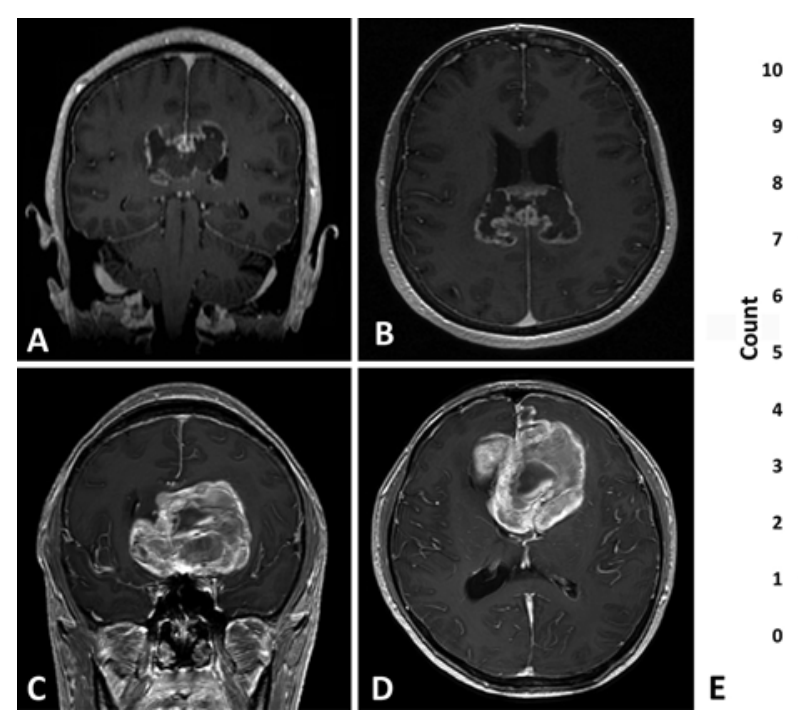

Tumor Volume Distribution

FIG. 1. Characteristics of butterfly glioma demonstrated on coronal and axial postgadolinium T1-weighted MRI for two separate patients. One patient (A-B) had a bGBM that spanned both hemispheres and was centered at the splenium of the CC. The other patient (C-D) had a bGBM in the frontal lobes that invaded the genu of the CC. Dot plot (E) depicting hemispheric volume distribution of bGBM as determined by the percentage of tumor volume in the hemisphere with the maximal tumor burden relative to total tumor volume.

patients were independent with regard to their functional status, with a median KPS score of 80 (range 20-90).

\section{Tumor Characteristics}

Tumor-specific characteristics of butterfly glioma are summarized in Tables 2 and 3. The most common sites for bGBM were the frontal (64.1\%) and parietal lobes (15.4\%). The bGBMs invaded the CC most commonly at the genu (48.7\%) and splenium (28.2\%). There was only 1 case (2.6\%) in which the bGBM invaded the CC at the rostrum. Volumetric analysis showed a median tumor volume of $39.5 \mathrm{~cm}^{3}$ with a range of $10.5-178.3 \mathrm{~cm}^{3}$. The volume of tumor in each cerebral hemisphere was used to determine in which hemisphere the maximal tumor burden was located. Twenty-one cases (53.8\%) had a higher tumor burden in the right hemisphere, while 17 cases $(38.5 \%)$ had a higher tumor burden in the left hemisphere. Three cases (7.7\%) were found to have an equal distribution of tumor burden in the two hemispheres. Volumetric analysis was used to calculate the percentage of tumor volume in the hemisphere with the maximal tumor burden relative to total tumor volume (Table 2 and Fig. 1E). Table 3 displays molecular profile of butterfly gliomas in our study stratified based on treatment approach of biopsy versus tumor resection.

TABLE 1. Clinical features of bGBM patients stratified by treatment approach of biopsy versus resection

\begin{tabular}{|c|c|c|c|c|}
\hline Characteristic & All Cases & Biopsy Cases & Resection Cases & $p$ Value \\
\hline No. of cases & 39 & 25 & 14 & \\
\hline Mean age at diagnosis in yrs (range) & $57.8(20-83)$ & $63.9(24-80)$ & $51.2(20-83)$ & 0.011 \\
\hline $\operatorname{Sex}($ no. $[\%])$ & & & & 0.32 \\
\hline Male & $23(59.0)$ & $13(52)$ & $10(71.4)$ & \\
\hline Female & $16(41.0)$ & $12(48)$ & $4(28.6)$ & \\
\hline Median KPS at diagnosis [IQR] & $80[60-90]$ & $70[60-80]$ & 90 [80-90] & 0.003 \\
\hline \multicolumn{5}{|l|}{ Symptoms at presentation (no. [\%]) } \\
\hline Headache & $19(48.7)$ & $12(63.2)$ & $7(36.8)$ & \\
\hline Confusion/memory loss & $13(33.3)$ & $9(69.2)$ & $4(30.8)$ & \\
\hline Seizure & $9(23.1)$ & $4(44.4)$ & $5(55.6)$ & \\
\hline Visual deficits & $8(20.5)$ & $5(62.5)$ & $3(37.5)$ & \\
\hline Nausea \& vomiting & $6(15.4)$ & $3(50)$ & $3(50)$ & \\
\hline Language deficits & $3(7.7)$ & $2(66.7)$ & $1(33.3)$ & \\
\hline Median preop tumor vol in $\mathrm{cm}^{3}[\mathrm{IQR}]$ & $39.5[27.2-55.4]$ & $37.6[26.2-53.8]$ & $43.7[33.8-70.2]$ & 0.26 \\
\hline
\end{tabular}

\footnotetext{
* Biopsy versus resection.
} 
TABLE 2. Tumor-specific features of bGBM

\begin{tabular}{lc}
\hline \multicolumn{1}{c}{ Feature } & Value \\
\hline Tumor location (no. [\%]) & $25(64.1)$ \\
\hline Frontal lobe & $6(15.4)$ \\
\hline Parietal lobe & $3(7.7)$ \\
\hline Temporoparietal lobe & $5(12.8)$ \\
\hline Other (frontoparietal, frontotemporal) & \\
\hline Site of CC invasion (no. [\%]) & $19(48.7)$ \\
\hline Genu & $5(12.8)$ \\
\hline Body & $11(28.2)$ \\
\hline Splenium & $4(10.3)$ \\
\hline Other (>1 area of CC) & $21(53.8)$ \\
\hline Hemisphere w/ max tumor burden (no. [\%]) & $15(38.5)$ \\
\hline Rt & $3(7.7)$ \\
\hline Lt & $67.1 \%$ \\
\hline Co-dominance & \\
\hline Median vol distribution in hemisphere w/ highest \\
tumor burden
\end{tabular}

\section{Treatment Approach and Survival Outcome}

Analysis of the treatment approach revealed 14 (35.9\%) cases that had undergone resection and 25 (64.1\%) cases that had undergone biopsy (Table 4). We did not find a statistically significant correlation between preoperative tumor volume and undergoing resection $(r=0.114, p=$ $0.5)$. Patients undergoing resection had a mean $67.5 \%$ of tumor on the dominant side (range 52.0\%-93.7\%), while patients undergoing biopsy had a mean $75.3 \%$ of tumor on the dominant side (range 52.1\%-98.1\%; $\mathrm{p}=0.07$ ). The median KPS score at the time of diagnosis in biopsy patients was 70 compared to 90 in the resection patients ( $\mathrm{p}$ $=0.003$ ). Median overall survival for the bGBM cohort was 3.2 months from diagnosis with a range of 0.3-25.9 months. The overall survival rates at 6,12 , and 18 months were $38.1 \%, 23.8 \%$, and $9.5 \%$, respectively. Univariate analysis demonstrated preoperative tumor volume to significantly impact survival, as expected, with a larger tumor volume associated with poorer survival $(r=-0.29, p$ $=0.03$; see Table 6 ).

\section{Biopsy Cohort}

Among the patients who underwent biopsy, 9 (36\%)
TABLE 4. Treatment approach to and survival outcome characteristics of bGBM

\begin{tabular}{lc}
\hline \multicolumn{1}{c}{ Element } & Specification \\
\hline Median overall survival in mos [IQR] & $3.20[1.80-9.45]$ \\
\hline 6-mo survival rate & \\
\hline Resection cases & $71.4 \%$ \\
\hline Biopsy cases & $20 \%$ \\
\hline 12-mo survival rate & $57.1 \%$ \\
\hline Resection cases & $8 \%$ \\
\hline Biopsy cases & $21.4 \%$ \\
\hline 18-mo survival rate & $4 \%$ \\
\hline Resection cases & $14(35.9)$ \\
\hline Biopsy cases & $8(57.14)$ \\
\hline Treatment approach (no. [\%]) & $25(64.1)$ \\
\hline Resection & $9(36)$ \\
\hline Resection + adjuvant chemoradiation & $5(20)$ \\
\hline Biopsy & \\
\hline Biopsy + adjuvant chemoradiation & $1(7.14)$ \\
\hline Biopsy + adjuvant radiation therapy & $83.04 \%(44.2 \%-100 \%)$ \\
\hline Resection outcomes & $62.7 \%(0 \%-100 \%)$ \\
\hline Median total EOR (range) & $90(60-90)$ \\
\hline Median CC EOR (range) & $80-90)$ \\
\hline Median preop KPS score (range) & \\
\hline Median postop KPS score (range) & \\
\hline Cases w/ immediate postop deficits (no. \\
$[\%])$
\end{tabular}

received adjuvant chemoradiation therapy in accordance with the Stupp protocol ${ }^{12}$ and $5(20 \%)$ patients received adjuvant radiation therapy (Table 4). The radiation therapy protocol involved focal fractionated external beam radiation in $78.6 \%$ of cases over a 6 -week period with a total radiation dose of $60 \mathrm{~Gy}$. Additionally, $14.3 \%$ of patients received fractionated whole-brain radiation with a total radiation dose of $30 \mathrm{~Gy}$. There were 11 patients (44\%) who underwent biopsy without any additional treatments. Among these patients were $6(24 \%)$ who were recommended for palliative care; the remaining 5 (20\%) patients died shortly after diagnosis.

\section{Resection Cohort}

For patients who underwent resection, the mean EOR was $81.7 \%$ (range $44.2 \%-100 \%$ ), with $57.1 \%$ of patients

TABLE 3. Molecular/genetic profile of bGBM cases that underwent tumor resection versus biopsy

\begin{tabular}{lcccccc}
\hline Procedure & EGFR Amplification & PTEN Deletion & TP53 Mutation & IDH1/2 Mutation & MGMT Methylation & ATRX Mutation \\
\hline Biopsy & $4 / 6$ & $3 / 8$ & $2 / 5$ & $1 / 10$ & $1 / 3$ & Not tested \\
\hline Resection & $4 / 11$ & $1 / 3$ & $2 / 3$ & $0 / 3$ & $0 / 2$ & Not tested \\
\hline
\end{tabular}

The denominator indicates the total number of cases analyzed, and the numerator indicates the number of cases that were positive for the corresponding genetic analysis. 
TABLE 5. Summary of immediate postoperative deficits and their outcome in patients who underwent resection

\begin{tabular}{cc}
\hline Case No. & Description; Outcome \\
\hline 1 & $\begin{array}{c}\text { Rt-sided hemiplegia, rt-sided neglect, slurred speech; } \\
\text { slight improvement but persistent deficit }\end{array}$ \\
\hline 2 & Blurred vision, seizure; improved to baseline levels \\
\hline 3 & Lt-sided neglect, It-sided weakness, apraxia; lost to FU \\
\hline 4 & $\begin{array}{c}\text { Expressive aphasia, perceptual motor difficulties, mild rt } \\
\text { visual field cut; improved to baseline levels }\end{array}$ \\
\hline
\end{tabular}

$\mathrm{FU}=$ follow-up.

undergoing unilateral resection of the dominant hemisphere and $42.9 \%$ undergoing bilateral resection. Our analysis showed that $57.1 \%, 21.4 \%$, and $21.4 \%$ of patients received adjuvant chemoradiation therapy, chemotherapy, and radiation therapy, respectively. And adjuvant chemoradiation therapy followed the Stupp protocol in $87.5 \%$ of cases. Moreover, all cases with adjuvant radiation therapy involved fractionated external beam radiation. The results of our analysis of patients who underwent resection are summarized in Table 4. The median KPS score for patients decreased from 90 preoperatively to 80 postoperatively $(\mathrm{p}=0.7)$. The median EOR was $83.0 \%$ with an IQR $75.6 \%-93.1 \%$. We identified 4 patients $(28.6 \%)$ with immediate postoperative neurological deficits (Table 5). There was improvement to baseline levels in 2 patients by the time each patient was seen for their first followup after discharge. One patient was lost to follow-up, and no additional information on their clinical recovery is known. One patient, despite a slight improvement in symptoms, suffered from persistent neurological deficits. Cox regression survival analysis of patients with immediate postoperative deficits compared to the patients with no postoperative deficits revealed no statistically significant differences between the two groups (HR 2.154, p = 0.282 ). With respect to the amount of $C C$ resected, we found a median $62.7 \%$ EOR involving 12 cases, while the 2 remaining cases only had hemispheric tumor resection (Table 4). A total of 3 cases had complete resection of the $\mathrm{CC}$ component of the tumor and 9 cases of partial resection of the $\mathrm{CC}$ portion of the tumor.

\section{Prognostic Factors}

Univariate analysis revealed that age, KPS score at diagnosis, preoperative tumor volume, and EOR for patients undergoing resection significantly impacted survival (Table 6). Additionally, we found that tumors whose volume distribution was predominantly confined to one hemisphere were associated with better survival than tumors with equal volume distribution in both hemispheres $(\mathrm{r}=0.3, \mathrm{p}=0.05)$. Postoperative tumor volume was also found to significantly impact survival, with a smaller residual tumor volume associated with better survival $(\mathrm{r}=$ $-0.565, p=0.035$ ). Patient sex was found not to impact survival in the univariate analysis. Additionally, the median EOR for the CC component of the tumor was not associated with better survival in the univariate analysis $(\mathrm{r}=0.284, \mathrm{p}=0.324)$. A preoperative age $\geq 70$ years was found to confer a poor prognosis (HR 3.14, $p=0.003$ ) in a Cox regression analysis. Figure $2 \mathrm{~A}$ displays the impact of preoperative age on survival outcomes in Kaplan-Meier survival curves. Patients whose age was $\geq 70$ years had a median overall survival of 2.04 months (IQR 1.62-6.97 months) compared to patients whose age was $<70$ years with a median overall survival of 4.60 months (IQR 1.99-16.3 months). Treatment approach significantly affected survival; patients who underwent tumor resection had a better prognosis than those who underwent biopsy (HR 0.37, $\mathrm{p}=0.009$ ). Median overall survival for patients who underwent biopsy was 2.53 months in comparison to 14.06 months for those who underwent resection. The Kaplan-Meier survival curve in Fig. 2B demonstrates the significant impact of treatment approach on survival. Additionally, the minimum EOR to observe a survival benefit was found to be $86 \%$ (HR 0.054, $\mathrm{p}=0.03$ ) with a median overall survival of 17.7 months for patients whose EOR > $86 \%$ compared to 2.43 months for patients whose EOR $\leq 86 \%$. The most aggressive treatment approach in our cohort involved tumor resection and combined chemoradiation therapy. Our survival analysis showed that patients receiving this treatment had a better prognosis than the patients who did not receive one of the three treatment modalities ( $\mathrm{HR}=0.34,16.8$ vs 3.17 months, respectively, $\mathrm{p}=0.015$ ). Kaplan-Meier survival curve in Fig. $2 \mathrm{C}$ displays treatment approach as a prognostic factor, with the

TABLE 6. Univariate and multivariate analyses demonstrating the impact of various clinical features on survival

\begin{tabular}{|c|c|c|c|c|c|c|c|c|c|}
\hline Analysis & Age & Sex & $\begin{array}{l}\text { KPS at } \\
\text { Dx }\end{array}$ & $\begin{array}{c}\text { Preop } \\
\text { Tumor Vol }\end{array}$ & $\begin{array}{l}\text { Biopsy vs } \\
\text { Resection }\end{array}$ & $\begin{array}{l}\text { Total } \\
\text { EOR }\end{array}$ & $\begin{array}{c}\text { CC } \\
\text { EOR }\end{array}$ & $\begin{array}{l}\text { Tumor Burden } \\
\text { Distribution }\end{array}$ & $\begin{array}{l}\text { Postop } \\
\text { Tumor Vol }\end{array}$ \\
\hline \multicolumn{10}{|l|}{ Univariate } \\
\hline Pearson correlation coefficient & -0.40 & 0.05 & 0.40 & -0.29 & 0.547 & 0.73 & 0.284 & 0.30 & -0.565 \\
\hline$p$ value & 0.02 & 0.76 & 0.01 & 0.03 & $<0.001$ & 0.003 & 0.324 & 0.05 & 0.04 \\
\hline \multicolumn{10}{|l|}{ Multivariate } \\
\hline Range risk ratio & 3.85 & - & 0.253 & 0.138 & 0.34 & - & - & - & - \\
\hline Lower 95\% Cl & 0.751 & - & 0.043 & 0.014 & 0.12 & - & - & - & - \\
\hline Upper 95\% Cl & 22.5 & - & 1.86 & 0.99 & 0.84 & - & - & - & - \\
\hline Likelihood ratio & 2.58 & - & 1.90 & 3.89 & 5.48 & & & & \\
\hline$p$ value & 0.11 & - & 0.168 & 0.048 & 0.019 & - & - & - & - \\
\hline
\end{tabular}

Dx $=$ diagnosis. 

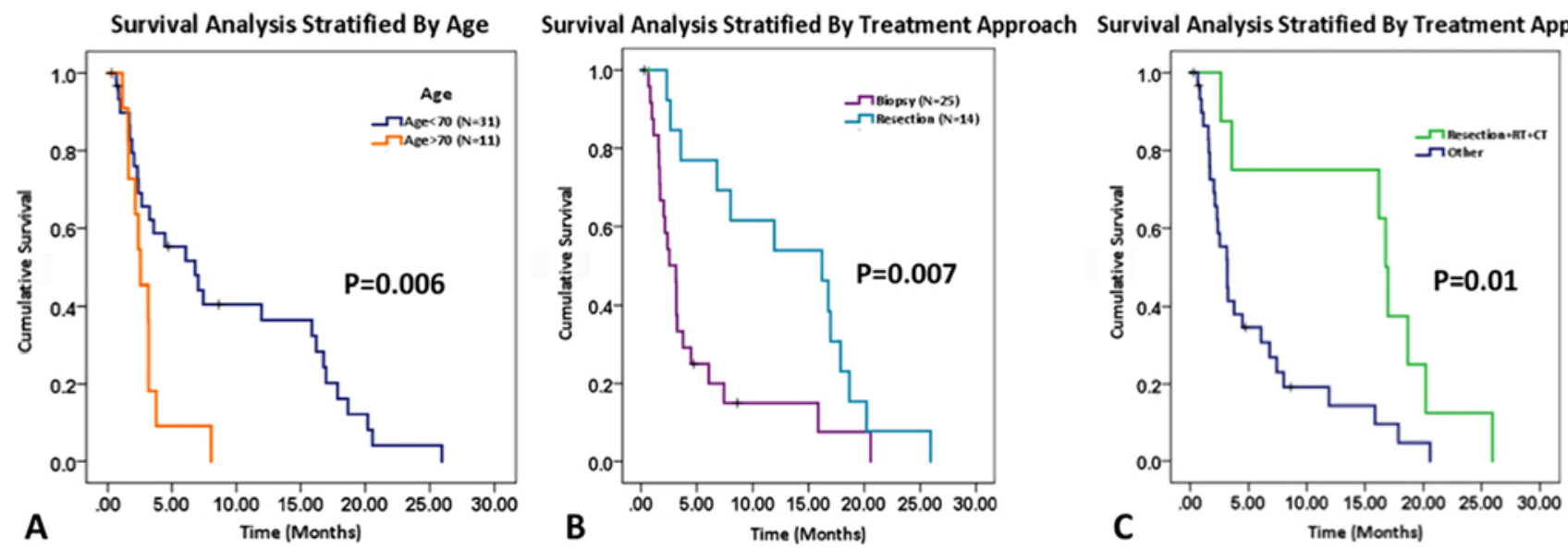

FIG. 2. Age and treatment approach are significant prognostic factors in patients with butterfly glioma. Kaplan-Meier survival curve stratified by patient age (A), treatment approach of biopsy versus resection (B), and combination of resection and chemoradiation therapy versus any other treatment approach (C).

combination of resection and chemoradiation conferring a better prognosis than any other therapeutic approach. On multivariate analysis, preoperative tumor volume and the treatment option of resection versus biopsy were found to impact survival independent of other prognostic factors including patient age and KPS score at the time of diagnosis (Table 6) with a larger tumor volume and undergoing biopsy conferring a worse prognosis.

\section{Discussion}

While aggressive resection has been shown to improve survival for patients with GBM, some tumors, such as butterfly gliomas, have historically been thought of as poor candidates for surgery given the high risk of damage to critical anatomical structures and the resultant devastating neurological deficits. More recently, advances in operative techniques and intraoperative cortical and subcortical mapping have made these lesions safer to resect. ${ }^{6}$ In the present retrospective analysis, we report an improvement in survival for patients who underwent resection versus biopsy alone (14.06 vs 2.53 months, respectively), with EOR also favorably impacting survival. Patients who underwent surgery were more likely to receive adjuvant chemotherapy and radiation treatment. Additionally, although approximately a third of patients had a postoperative deficit, the majority of these deficits were transient and improved to baseline levels, and the KPS score was not significantly changed postoperatively ( 90 preoperative vs 80 postoperative, $p=0.70$ ). Unsurprisingly, older patients ( $\geq 70$ years old) were more likely to succumb to their disease earlier than the younger patients. While upfront palliation or presumptive treatment is an option for these patients, obtaining enough tissue for extensive molecular markers and genetic testing can guide treatment decisions, aid in prognosis counseling, identify potential targeted therapies, and determine eligibility for clinical trials. To our knowledge, this is the first study to explore EOR within the CC itself. We found that EOR of the CC component of the GBM was $62.7 \%$ compared to total EOR, which was $83.04 \%$, but that EOR of the callosal component did not impact survival.
These results are consistent with those of other series in the literature ${ }^{6-8,11}$ (Table 7). In our study, the average EOR was slightly less than that in other reports, though it still eclipsed the $>65 \%$ EOR benchmark set by Chaichana et al. ${ }^{7}$ Additionally, we did not restrict resection to lesions that crossed the anterior $\mathrm{CC}$, a group that has previously been excluded from some case series. The low rate of permanent complications makes surgery a viable option for patients given the unfortunate natural history of the disease. The minimum EOR to observe a survival benefit was $86 \%$ in our cohort. This finding is within the range of the minimum EOR needed to impact survival as reported in previous studies of GBMs independent of anatomical location $^{5,9}$ and is consistent with the fact that no studies to date have identified aggressive biological features specific to bGBMs, suggesting that their historical poor prognosis stems from a lack of surgical aggressiveness in the CC. Our work also suggested that these tumors are similar to GBMs in general in that there may be an EOR threshold to achieve in order to maximize the survival benefit of resection and that crossing this EOR threshold for the entire bGBM is more important than the EOR achieved within the callosal component itself. Although the inherent biases of these retrospective case series should be considered when evaluating their conclusions, the consistent nature of the findings adds support to our results.

Given these findings, even patients with large bGBMs can safely undergo tumor resection if they have relatively minor symptoms and appear to be good surgical candidates otherwise. Any surgical approach needs to avoid injuring critical neural and vascular structures such as the caudate, cingulum, septal nuclei, and anterior cerebral artery branches. Serious complications such as extraaxial hemorrhage and large vessel territory infarcts, which have been reported in the literature,$^{5}$ were not seen in our cohort.

Other reports in the literature have employed different operative techniques for resection of these lesions. For example, in the series by Burks et al., recent operations were performed with the patients awake, and subcortical mapping of the cingulum and cingulate cortex was used 
TABLE 7. Summary of recent reports on surgical treatment for high-grade butterfly gliomas

\begin{tabular}{|c|c|c|c|c|c|}
\hline Authors \& Year & $\begin{array}{l}\text { No. of Pts } \\
\text { Treated w/ Op }\end{array}$ & $\begin{array}{l}\text { Mean Age } \\
\quad(y r s)\end{array}$ & $\begin{array}{c}\text { Overall } \\
\text { Complication Rate }\end{array}$ & $\begin{array}{c}\text { Survival for bGBM Pts } \\
\text { Treated w/ Resection (mos)* }\end{array}$ & $\begin{array}{l}\text { Survival for bGBM Pts } \\
\text { w/o Resection (mos)* }\end{array}$ \\
\hline Dziurzynski et al., 2012 & 11 & 59 & - & 7.3 & 1.3 \\
\hline Chaichana et al., 2014 & 29 & 61.7 & - & 7.0 & 3.5 \\
\hline Burks et al., 2017 & 40 & $52 \& 45 \dagger$ & $22.5 \%$ & 15.0 & - \\
\hline Opoku-Darko et al., 2017 & 9 & 56.9 & $22.2 \%$ & 7.8 & 2.8 \\
\hline Present study & 14 & 57.8 & $28.6 \%$ & 14.1 & 2.5 \\
\hline
\end{tabular}

to identify high-order functions in an attempt to minimize the prevalence of abulia and akinetic medial frontal lobe syndromes. ${ }^{6}$ Alternatively, Opoku-Darko et al. preferred general anesthesia given the length and complexity of the operation. ${ }^{11}$ We report a similar experience, with all of our patients having undergone general anesthesia.

This study is limited by its retrospective nature and small sample size. Age and KPS score both preoperatively and at diagnosis were different in the patients who underwent biopsy compared to those who underwent resection, which likely influenced the surgeon's decision to offer less aggressive treatment in the former group. Given the paucity of literature regarding the resection of bGBM, this study provides much needed evidence to help guide treatment decision-making for patients with this disease. Our results should be replicated and validated with prospective studies. Further prospective studies exploring whether the type of surgical approach (for example, awake surgery with subcortical mapping versus surgery with general anesthesia) influences EOR, postoperative deficits, and overall survival are needed to further characterize the best technique for cytoreduction of these tumors. Additionally, comparing quality of life and psychological questionnaires for patients who undergo resection with those for patients who undergo either biopsy only or palliative treatment can further quantify the morbidity and effect of these procedures versus the natural history of the disease. Given that patients who undergo aggressive surgery are likely to initially spend more time in the intensive care unit and hospital, if the improvement in survival is not associated with a correspondent improvement in quality of life, then it is possible that patients may opt for less aggressive treatment that is better aligned with their individual preferences. Finally, more minimally invasive techniques, such as laserinduced interstitial therapy (LITT), ${ }^{10}$ and new technologies, such as intraoperative MRI, may improve the safety of resection ${ }^{9}$ or provide an alternative or adjuvant to open surgical cytoreduction and enhance the extent of tumor removal and survival for patients, particularly those with small-volume tumors. ${ }^{4}$

\section{Conclusions}

In summary, bGBM occurs in a rare anatomical location for GBMs and makes up a small percentage of highgrade glioma cases. This retrospective study suggests that resection can be safely performed in patients and that cy- toreduction of these tumors can lead to an improvement in overall survival. Selecting patients as surgical candidates is dependent on the operating neurosurgeon, but factors including age and functional status at the time of diagnosis (that is, KPS) appear to significantly affect overall survival and should be taken into consideration in the treatment decision-making process. Should these results be confirmed with a prospective analysis, future work quantifying the quality of life for patients who undergo surgery, exploring minimally invasive cytoreductive techniques, and characterizing patients most appropriate for resection is needed to help guide treatment decisions for both physicians and patients.

\section{References}

1. Agrawal A: Butterfly glioma of the corpus callosum. J Cancer Res Ther 5:43-45, 2009

2. Almeida JP, Chaichana KL, Rincon-Torroella J, QuinonesHinojosa A: The value of extent of resection of glioblastomas: clinical evidence and current approach. Curr Neurol Neurosci Rep 15:517, 2015

3. Balaña C, Capellades J, Teixidor P, Roussos I, Ballester R, Cuello M, et al: Clinical course of high-grade glioma patients with a "biopsy-only" surgical approach: a need for individualised treatment. Clin Transl Oncol 9:797-803, 2007

4. Beaumont TL, Mohammadi AM, Kim AH, Barnett GH, Leuthardt EC: Magnetic resonance imaging-guided laser interstitial thermal therapy for glioblastoma of the corpus callosum. Neurosurgery [epub ahead of print], 2018

5. Bloch O, Han SJ, Cha S, Sun MZ, Aghi MK, McDermott MW, et al: Impact of extent of resection for recurrent glioblastoma on overall survival: clinical article. J Neurosurg 117:1032-1038, 2012

6. Burks JD, Bonney PA, Conner AK, Glenn CA, Briggs RG, Battiste JD, et al: A method for safely resecting anterior butterfly gliomas: the surgical anatomy of the default mode network and the relevance of its preservation. J Neurosurg 126:1795-1811, 2017

7. Chaichana KL, Jusue-Torres I, Lemos AM, Gokaslan A, Cabrera-Aldana EE, Ashary A, et al: The butterfly effect on glioblastoma: is volumetric extent of resection more effective than biopsy for these tumors? J Neurooncol 120:625-634, 2014

8. Dziurzynski K, Blas-Boria D, Suki D, Cahill DP, Prabhu SS, Puduvalli V, et al: Butterfly glioblastomas: a retrospective review and qualitative assessment of outcomes. J Neurooncol 109:555-563, 2012

9. Lara-Velazquez M, Al-Kharboosh R, Jeanneret S, VazquezRamos C, Mahato D, Tavanaiepour D, et al: Advances in 
brain tumor surgery for glioblastoma in adults. Brain Sci 7:E166, 2017

10. Mohammadi AM, Hawasli AH, Rodriguez A, Schroeder JL, Laxton AW, Elson P, et al: The role of laser interstitial thermal therapy in enhancing progression-free survival of difficult-to-access high-grade gliomas: a multicenter study. Cancer Med 3:971-979, 2014

11. Opoku-Darko M, Amuah JE, Kelly JJP: Surgical resection of anterior and posterior butterfly glioblastoma. World Neurosurg 110:e612-e620, 2018

12. Parsa AT, Wachhorst S, Lamborn KR, Prados MD, McDermott MW, Berger MS, et al: Prognostic significance of intracranial dissemination of glioblastoma multiforme in adults. J Neurosurg 102:622-628, 2005

13. Sanai N, Polley MY, McDermott MW, Parsa AT, Berger MS: An extent of resection threshold for newly diagnosed glioblastomas. J Neurosurg 115:3-8, 2011

14. Siddiqui J, Krishnan AS: Butterfly glioma. N Engl J Med 378:281, 2018

15. Steltzer KJ, Sauvé KI, Spence AM, Griffin TW, Berger MS: Corpus callosum involvement as a prognostic factor for patients with high-grade astrocytoma. Int J Radiat Oncol Biol Phys 38:27-30, 1997

16. Stupp R, Mason WP, van den Bent MJ, Weller M, Fisher B, Taphoorn MJB, et al: Radiotherapy plus concomitant and adjuvant temozolomide for glioblastoma. N Engl J Med 352:987-996, 2005

\section{Disclosures}

The authors report no conflict of interest concerning the materials or methods used in this study or the findings specified in this paper.

\section{Author Contributions}

Conception and design: Aghi, Dayani. Acquisition of data: Dayani, Bonte. Analysis and interpretation of data: Aghi, Dayani. Drafting the article: Aghi, Dayani, Young. Critically revising the article: Aghi, Dayani, Young. Reviewed submitted version of manuscript: Aghi, Dayani, Young, Chang, Theodosopoulos, McDermott, Berger. Approved the final version of the manuscript on behalf of all authors: Aghi. Statistical analysis: Dayani. Study supervision: Aghi, Dayani.

\section{Correspondence}

Manish K. Aghi: University of California, San Francisco, CA. manish.aghi@ucsf.edu 